\title{
Communications
}

\section{Surface Morphology Evolution of Chemical Vapor-Deposited Tungsten Films on Si(100)**}

\author{
By Luis Vázquez,* Roberto C. Salvarezza, \\ Ezequiel Albano, Alejandro J. Arvia, \\ Alberto Hernández Creus, Roland A. Levy, and \\ José M. Albella
}

Chemical vapor deposition (CVD) is used to prepare research-grade heterostructures and to produce the majority of industrially important thin films. ${ }^{[1]}$ In particular, CVD tungsten films are used for many technological applications. $^{[2,3]}$ In CVD an external source maintains a fixed concentration of reactant molecules at a distance above the film surface. ${ }^{[4]}$ Then, gas diffusion drives the molecules through the diffusion layer ${ }^{[2]}$ towards the film surface. At the film interface a reaction must occur before new material is incorporated into the solid. Kinetic studies show that two growth regimes are usually present in CVD. At a low deposition temperature (low rate, regime I) the kinetics is controlled by the surface reaction, whereas at a high temperature (high rate, regime II), mass transport of reactants to, or reaction products from, the surface is the rate-controlling step. ${ }^{[1]}$

For many applications, it is convenient to be able to control the dynamics and structure of the growing film surface, which are mainly determined by the film growth mechanism. Thus, several models of CVD have been proposed in relation to the interface growth dynamics that include destabilizing and stabilizing factors. ${ }^{[4-7]}$ The predictions of these models have been difficult to check due to the lack of systematic experimental results in the evolution of CVD films, specially on the nanometer/micrometer scale where typical morphological instabilities occur. ${ }^{[4]}$

[*] Dr. L. Vázquez, Dr. J. M. Albella

Instituto de Ciencia de Materiales de Madrid (CSIC)

Cantoblanco, E-28049 Madrid (Spain)

Dr. R. C. Salvarezza, Dr. E. Albano, A. J. Arvia

Instituto de Investigaciones Fisicoquímicas Teóricas y

Aplicadas (CONICET)

Sucursal 4, Casilla de Correo 16, 1900 La Plata (Argentina)

Dr. A. Hernández Creus

Departamento de Química Física

Universidad de La Laguna

E-38071 La Laguna, Tenerife (Spain)

Dr. R. A. Levy

New Jersey Institute of Technology

University Heights, Newark, NJ 07102 (USA)

[**] This work was partially supported by the CSIC-CONICET cooperation program, by a grant from the NASA New Jersey Space Grant Consortium, and the Commission of the European Communities under contract ITDC-122.
The interface evolution can be followed by means of the dynamic scaling theory applied to surface profiles on different time scales. ${ }^{[8]}$ This theory predicts that the interface width (the root mean square roughness), $w(L, h)$, for a length $L$ and average thickness $h$, scales as $w(t) \propto t^{\mathrm{b}}$ when $t$ $\rightarrow 0$, whereas for $t \rightarrow \infty$, it scales as $w(L) \propto L^{a}$, the exponents $\mathrm{b}, \mathrm{a}$, and $z=\mathrm{a} / \mathrm{b}$ being the growth, roughness, and dynamic exponents, respectively, while $1 / z$ is known as the coarsening exponent. ${ }^{[9]}$ The dynamic scaling theory has already been applied to study polycrystalline films grown by evaporation $^{[10]}$ and electrodeposition. ${ }^{[11]}$

In this work, we present experimental data on the dynamics of $\mathrm{W}$ surfaces grown by low pressure CVD (LPCVD $)^{[1]}$ under regimes I and II.

The growth rate, $u$, versus $1 / T$ plot for our CVD system (Fig. 1) is similar to the standard Arrhenius plot for CVD, as it exhibits two regions with a crossover at $T=720 \mathrm{~K}$. For $T<720 \mathrm{~K}$, $u$ increases with $T$ and the growth kinetics is controlled by a thermally activated surface reaction (regime I). ${ }^{[2]}$ The activation energy, as calculated from the slope of the low temperature linear portion of the curve, was found to be equal to $60 \mathrm{~kJ} / \mathrm{mol}$. This value, which is lower than those reported for tungsten films $(67-73 \mathrm{~kJ} /$ $\mathrm{mol}),{ }^{[3]}$ may be the result of using a cold wall reactor system, where an accurate measurement of the deposition temperature is difficult, especially at low pressures. For $T>$ $720 \mathrm{~K}$, $u$ increases slightly with $T$ and the growth process is controlled by the rate at which the reactants are supplied to the growing film (regime II).

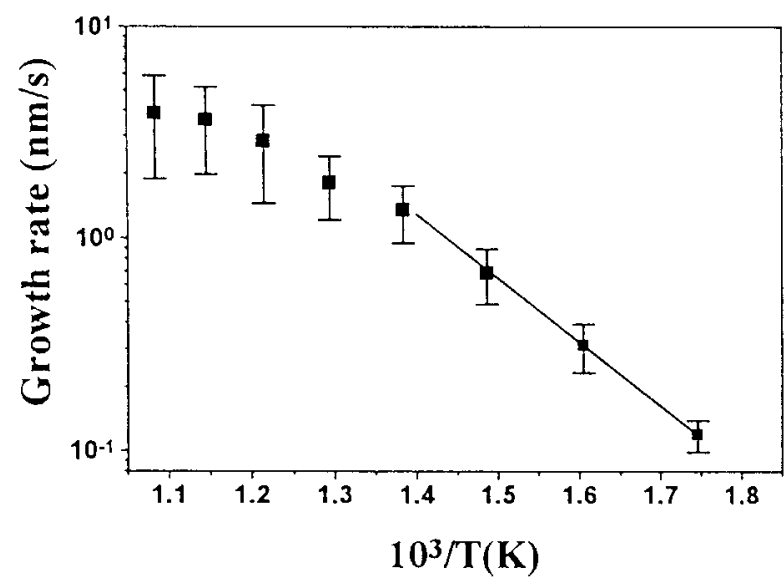

Fig. 1. Plot of u versus $1 / T$ for CVD W deposition. The straight line shows the thermally activated kinetic-limited growth regime (regime I). The high temperature region with a nearly constant growth rate corresponds to the mass transport-limited regime (regime II).

Typical STM images of $\mathrm{W}$ films grown at $T=623 \mathrm{~K}$ and $T=823 \mathrm{~K}$, and different deposition times $(t)$ are shown in Figure $2 \mathrm{a}-\mathrm{d}$. Both series of films become rougher and their 


\section{Chenjical Vapor$$
\text { Deposition }
$$

average grain size $(d)$ increases markedly as $t$ increases, but this effect is clearer for $\mathrm{W}$ films grown at a higher $T$ (Fig. 3). The value of $d$ can be evaluated directly from the STM images and the coarsening exponent, $1 / z$, can be estimated. Thus, the $d$ versus $t$ logarithmic plots lead to coarsening exponents $1 / z=0.43 \pm 0.09$ and $1 / z=0.61 \pm 0.09$ for regimes I and II, respectively (Fig. 3a). a)

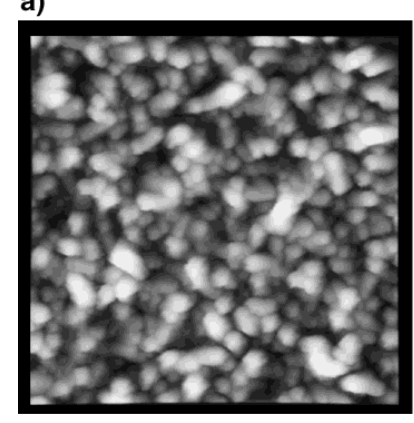

c)

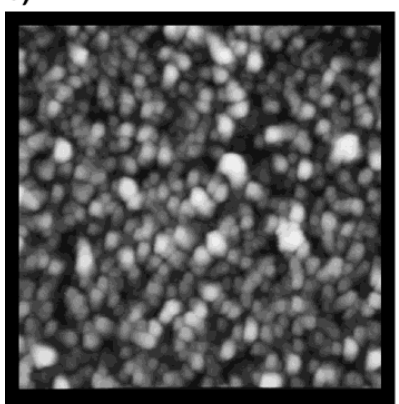

b)

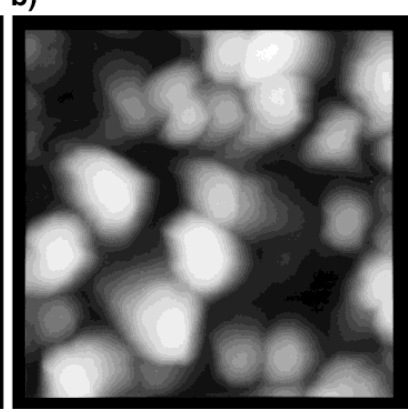

d)

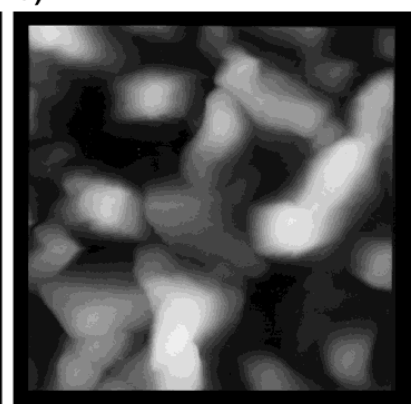

Fig. 2. a,b) Top view STM images $\left(4 \times 4 \mu \mathrm{m}^{2}\right)$ of CVD W films grown at $T=$ $623 \mathrm{~K}$ : a) $t=200 \mathrm{~s}$; b) $t=24000 \mathrm{~s}$. c,d) Top view STM images $\left(8 \times 8 \mu \mathrm{m}^{2}\right)$ of CVD W films grown at $T=823 \mathrm{~K}: \mathrm{c}) t=70 \mathrm{~s} ; \mathrm{d}) t=1800 \mathrm{~s}$.

The root mean square (rms) roughness, $w$, measured over $10 \times 10 \mu \mathrm{m}^{2}$ STM images, increases with $t$, but the increase is much faster for the $\mathrm{W}$ films grown at $T=823 \mathrm{~K}$ than for those grown at $T=623 \mathrm{~K}$. The $w$ versus $t$ logarithmic plots (Fig. 3b) yield straight lines with slopes $\mathrm{b}=0.37 \pm 0.09$ for $T$ $=623 \mathrm{~K}$ and $\mathrm{b}=0.54 \pm 0.09$ for $T=823 \mathrm{~K}$. However, in both cases, the grain surfaces remain smooth, indicating that surface diffusion operates at length scales close to $d$. It should be noted that the main mechanism of $\mathrm{W}$ surface relaxation, under our experimental conditions, should be surface diffusion because of the extremely low vapor pressure of $\mathrm{W}^{[12]}$

In practice, in CVD processing, it is necessary to deposit a film of given thickness and roughness. Since the growth rate in regime II is one order of magnitude larger than in regime $I$ it is important, then, to compare the roughness for films deposited in the two regimes but with the same thickness (i.e., different deposition times). This analysis can be made from Figure 3b, where it is evident that for low film thickness the roughness in the two regimes tends to be similar. However, as the film thickness increases the roughness of the film deposited in regime II increases faster than that of the film grown in regime I. This fact is a consequence of the larger b value obtained for regime II.

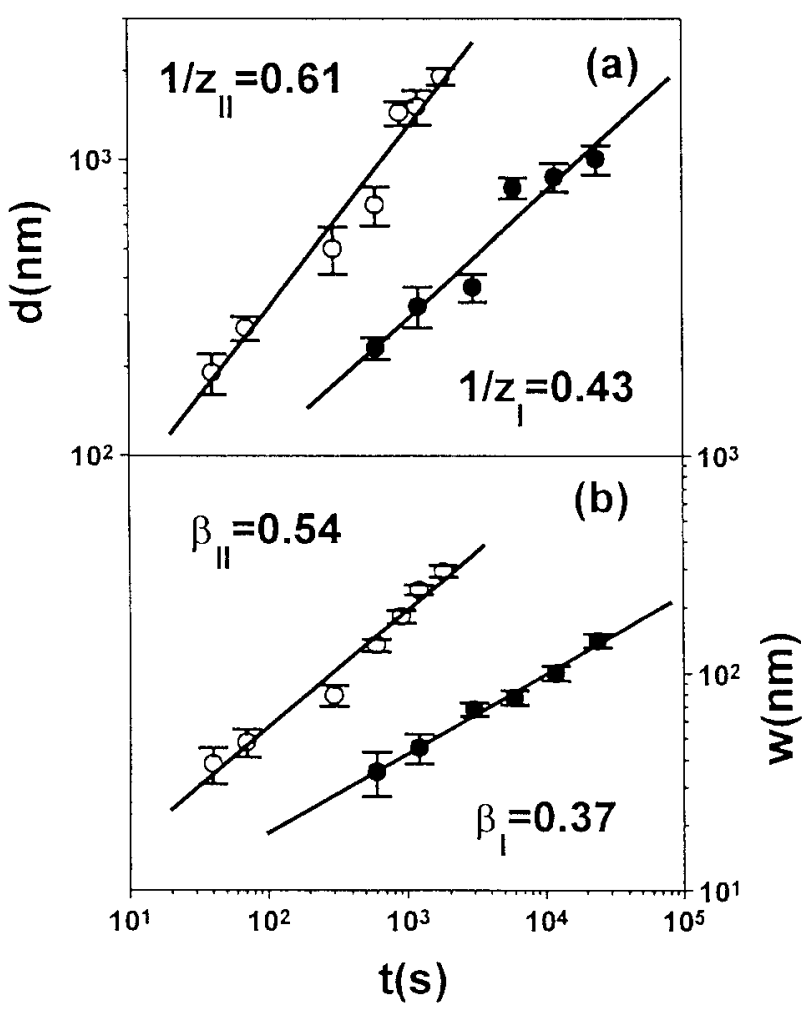

Fig. 3. Logarithmic plots of a) $d$ versus $t$ and b) $w$ versus $t$ for CVD W films deposited at $T=623 \mathrm{~K}$ (filled symbols) and $T=823 \mathrm{~K}$ (open symbols).

The $w$ versus $L$ logarithmic plots (Fig. 4a,b) obtained from the STM images ${ }^{[13]}$ show straight lines with a slope a $=0.84 \pm 0.05$, irrespective of $T$ and $t$ (see insets of Fig. 4). The values of both exponents were confirmed by evaluating the height-height correlation function from the STM images. Note that, because $w$ does not reach a steady-state roughness value in either regime, $a$ and $b$ must be considered as effective rather than true scaling exponents. ${ }^{[9]}$ However, the b/a ratio is close to the value of $1 / z$ obtained directly from the STM images.

For regime II, the value $\mathrm{b}>0.5$ indicates an unstable or marginally stable interface growth. ${ }^{[9]}$ The physical explanation of this instability is that a particle executing a random walk towards the growing surface, driven by the concentration field, has a higher probability of hitting an outward surface projection than of penetrating into a surface depression. This implies that the growth rate at outer surfaces is higher than at inner regions. ${ }^{[6]}$

For regime $\mathrm{I}, \mathrm{a}=0.84 \pm 0.05, \mathrm{~b}=0.37 \pm 0.09$, and $1 / z=$ $0.43 \pm 0.09$ are obtained. These values coincide with those predicted by growth models that include surface diffusion with energetic barriers at step edges $(a \approx 1$ and $0.25 \leq \mathrm{b} \leq$ $0.5,{ }^{[14-17]}$ as confirmed by three-dimensional Monte Carlo simulations). However, the existence of step-edge barriers for polycrystalline films with a random orientational distribution has been questioned because of the absence of welldefined terraces. ${ }^{[10]}$ Thus, other possible mechanisms explaining the values of the exponents could operate. STM 
images of W films (Fig. 2) suggest that the simplest description for the interface evolution of these polycrystalline films could be based on a model incorporating random deposition, surface diffusion, grain-grain competition, and intergranular mass transport.

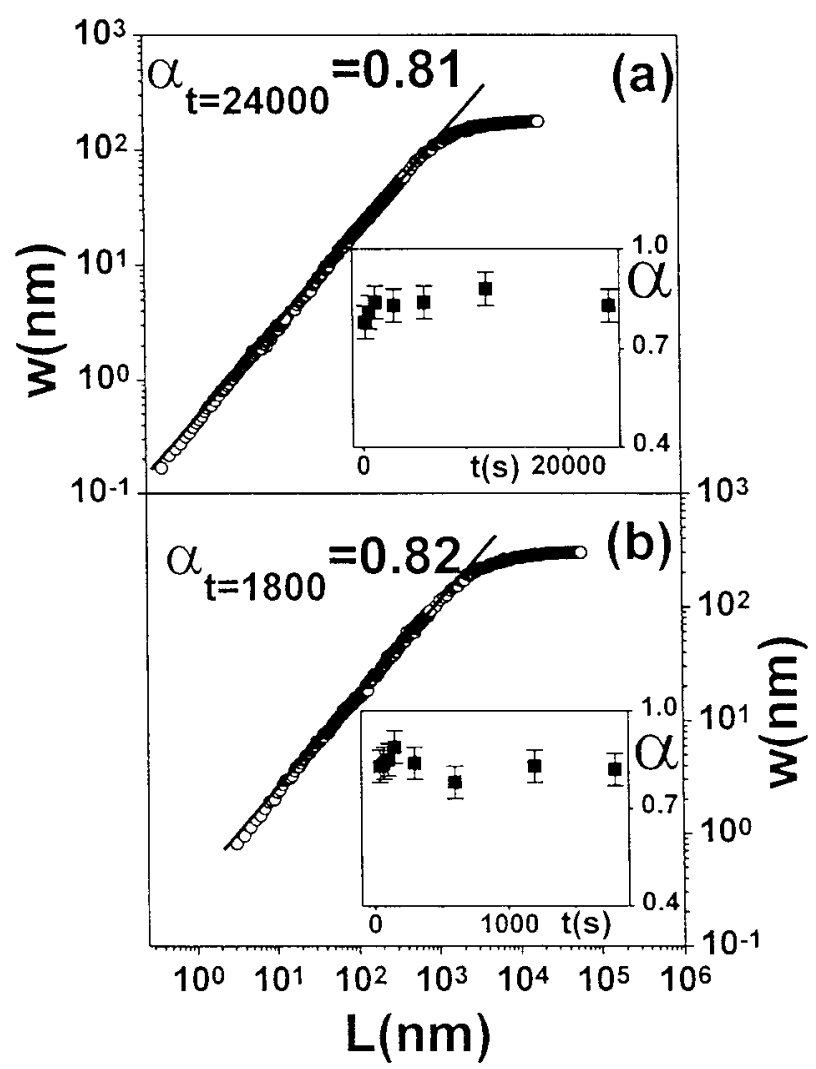

Fig. 4. Logarithmic plots of $w$ versus $L$ for a CVD W film deposited at a) $623 \mathrm{~K}, t=24000 \mathrm{~s}$ and b) $823 \mathrm{~K}, t=1800 \mathrm{~s}$. The insets show the a versus $t$ plots.

Thus, the growth of CVD W films has been simulated using a two-dimensional off-lattice substrate ${ }^{[9]}$ of side $M$ with a periodic boundary condition. Initially, nucleation centers with a density $r_{0}$ were randomly distributed on the substrate. In the submonolayer regime, grains, randomly oriented, grow by the capture of particles arriving by surface diffusion at grain edges and form intergranular gaps. After monolayer completion, grain growth proceeds according to a generalization of the rule for random deposition with surface diffusion to the nearest neighbor site. ${ }^{[9]}$ Particles arriving at the edges of higher grains are allowed to be attached, with probability $p_{\mathrm{s}}$, to higher grains shadowing sites of lower grains, while the probability that they relax and attach to lower grains is $1-p_{\mathrm{s}}$. Three-dimensional simulations of this model, performed for $0.05 \leq p_{\mathrm{s}} \leq 1$, lead to films where rapid coarsening takes place due to shadowing among smooth grains. These simulations lead, for $1 \geq p_{\mathrm{s}} \geq 0.05$, to $0.32 \pm 0.03 \leq 1 / z \leq 0.39 \pm 0.03$ and $0.52 \pm 0.07 \leq \mathrm{b} \leq 0.26 \pm 0.05$. The values of the exponents found for regime I are in the range predicted by this model. Thus, these results support the simultaneous contribution to the interface evolution in regime I of shadowing, which causes a rapid grain coarsening, and of mass transport between growing grains.

In conclusion, we have found that the interface evolution under regime I fulfills the predictions of a growth model for polycrystalline films, including random deposition, surface diffusion, shadowing among growing grains, and intergranular mass transport. For regime II, the tops of the crystals become more efficient than valleys at capturing the arriving flux of material, causing the interface to become extremely unstable with a high coarsening exponent.

\section{Experimental}

The $\mathrm{W}$ films investigated were synthesized by LPCVD in a SPECTRUM model 211, cold wall, single wafer, fully automated CVD reactor. Single crystal (100) silicon wafers with a rms roughness of $0.14 \mathrm{~nm}$, measured by atomic force microscopy, were used as substrates. The $\mathrm{W}$ films were synthesized at a pressure of 500 mTorr under constant $\mathrm{WF}_{6}(5 \mathrm{sccm})$ and $\mathrm{H}_{2}$ $(100 \mathrm{sccm})$ flow rates. LPCVD W depositions were performed at $623 \mathrm{~K}$ (regime I, average growth rate $u=0.35 \mathrm{~nm} \mathrm{~s}^{-1}$ ) and $823 \mathrm{~K}$ (regime II, $u=3$ $\mathrm{nm} \mathrm{s}^{-1}$ ). The films grown in either regime were polycrystalline with random orientation, as observed by X-ray diffraction.

The surfaces were imaged using a Nanoscope III Scanning Tunneling Microscope operating in ambient conditions with Pt-Ir tips. STM images were analyzed using the single-image dynamic scaling method [13] after fitting the instrument plane and applying a subtracting procedure [18].

Received: August 28, 1997 Final version: December 8, 1997

[1] W. Kern, in Microelectronic Materials (Ed: R. A. Levy), Kluwer Academic, Dordrecht, The Netherlands 1989, p. 203.

[2] J. Randall Creighton, J. E. Parmeter, Crit. Rev. Solid State Mater. Sci. 1993, 18,175 .

[3] C. M. McConica, K. Krishnamani, J. Electrochem. Soc. 1986, 133, 2542.

[4] G. S. Bales, A. C. Redfield, A. Zangwill, Phys. Rev. Lett. 1989, 62, 776.

[5] H. J. Viljoen, J. J. Thiart, V. Hlavacek, AIChE J. 1994, 40, 1032

[6] B. J. Palmer, R. G. Gordon, Thin Solid Films 1988, 158, 313; ibid. 1989, 177, 141.

[7] C. H. J. van den Brekel, A. K. Jansen, J. Cryst. Growth 1978, 43, 364.

[8] F. Family, T. Vicsek, Dynamics of Fractal Surfaces, World Scientific, Singapore 1991

[9] A. L. Barabasi, H. E. Stanley, Fractal Concepts in Growth Phenomena, Cambridge University Press, Cambridge 1995.

[10] J. H. Jeffries, J.-K. Zuo, M. M. Craig, Phys. Rev. Lett. 1996, 76, 4931.

[11] A. Iwamoto, T. Yoshinobu, H. Iwasaki, Phys. Rev. Lett. 1994, 72, 4025.

[12] CRC Handbook of Chemistry and Physics (Eds: D. R. Lide, H. P. R. Frederikse) CRC Press, Boca Raton, FL 1994.

[13] R. C. Salvarezza, L. Vázquez, P. Herrasti, P. Ocón, J. M. Vara, A. J. Arvia, Europhys. Lett. 1992, 20, 727.

[14] M. Siegert, M. Plischke, Phys. Rev. Lett. 1994, 73, 1517.

[15] Z. Zhang, J. Decht, H. Metiu, Phys. Rev. B 1993, 48, 4972

[16] P. Smilauer, D. D. Vvedensky, Phys. Rev. B 1995, 52, 14263

[17] A. Hernández Creus, P. Carro, R. C. Salvarezza, A. J. Arvia, Langmuir 1997, 13, 833.

[18] J. Krim, I. Heyvaert, C. van Haesendonck, Y. Bruynseraede, Phys. Rev. Lett. 1992, 70, 57 